\title{
The Effect of Fibre on Chronic Constipation in Adults: A Systematic Review
}

Symptoms of chronic constipation are common, with a prevalence of $\sim 14 \%$ in adults worldwide ${ }^{(1)}$. Although increasing fibre intake is universally accepted as a first-line treatment ${ }^{(2)}$, patient dissatisfaction is common. A systematic review is thus required in order to evaluate the quality of scientific evidence behind this management approach.

The aim was to assess the effect of fibre on chronic constipation in adults via a systematic review of randomised controlled trials (RCTs).

Following Cochrane and PRISMA ${ }^{(3)}$ recommendations, references were identified by searching 5 electronic databases, hand searching abstracts of 3 annual conferences, as well as scanning reference lists. Two reviewers independently assessed all studies identified, and performed data extraction and risk of bias assessment. Only RCTs reporting administration of fibre (carbohydrate polymers with $\geq 3$ monomers that are not hydrolysed by endogenous enzymes in the small intestine) in adults with chronic idiopathic constipation were included.

Of 916 records identified, only 7 RCTs were eligible (447 patients). Fibre supplements, administered in the form of powder, granules, yoghurt or milk, were: psyllium $(n=2)$, galacto-oligosaccharides (GOS: $n=2)$, inulin $(n=1)$, and mixtures of inulin/ resistant maltodextrin $(n=1)$, and inulin/partially hydrolysed guar gum $(n=1)$. Outcomes measured included stool frequency $(n=7)$, stool consistency $(n=4)$, stool weight $(n=3)$, faecal microbiota $(n=3)$ and adverse events $(n=2)$. Three out of 7 RCTs demonstrated a significant increase in stool frequency following fibre consumption compared to placebo. Stool consistency significantly improved following fibre consumption in 2 RCTs, while 2 RCTs failed to show an effect. One RCT demonstrated a significant increase in stool weight, but 2 RCTs did not. Bifidobacteria significantly increased in 2 out of 3 RCTs (inulin and one GOS intervention). No side effects were observed and no patient withdrew because of adverse events. Attrition bias was high amongst trials, while selection bias and performance bias were unclear due to inadequate reporting. Outcome data will undergo meta-analysis.

Current guidelines recommend the use of fibre as first-line treatment for constipation. However, this review shows that not all studies support its use in adults. Most studies suffer from small sample sizes and poor design with high risk of bias. The paucity of high quality data highlights the need for further large, methodologically rigorous RCTs.

1. Belsey J, Greenfield S, Candy D et al. (2010) Aliment Pharmacol Ther 31, 938-949.

2. Suares NC \& Ford AC (2011) Aliment Pharmacol Ther 33, 895-901.

3. Liberati A, Altman DG, Tetzlaff J et al. (2009) Ann Intern Med 151, 65-94. 